\title{
Turning THz Communications into Reality: Status on Technology, Standardization and Regulation
}

\author{
Thomas Kürner ${ }^{1}$ \\ ${ }^{1}$ Technische Universität Braunschweig, Institut für Nachrichtentechnik, Braunschweig, Germany
}

\begin{abstract}
Already a couple of years ago THz communications have not only become an attractive new research area on channel modeling but also triggered a couple of projects heading to develop appropriate technological solutions to enable the set-up of hardware demonstrators. Also discussions and activities in standardization and regulation already took off. In October 2017, IEEE published Std. IEEE 802.15.3d-2017 the worldwide first wireless communications standard operating in the $300 \mathrm{GHz}$ frequency band. In parallel to the standardization process activities at the ITU-R level targeting on the provision of an appropriate regulatory framework at the World Radio Conference 2019 (WRC-2019) via a dedicated agenda item have taken off. This paper provides a brief overview on the current status of the development of $\mathrm{THz}$ Communication systems focusing on recent results on advanced channel characterization at $300 \mathrm{GHz}$, hardware demonstrators operating in this frequency range, the past and current activities at IEEE 802 and the WRC 2019 preparations.
\end{abstract}

\section{INTRODUCTION}

The every-increasing need for wireless data rates will soon reach the order of $100 \mathrm{Gbit} / \mathrm{s}$ for a couple of applications. Among those applications are backhaul/fronthaul links required to connect $5 \mathrm{G}$ access points, complementary wireless links in data centers, kiosk downloading and even wireless links inside devices like cameras or video projectors [1]. The large amount of spectrum available beyond $275 \mathrm{GHz}$ makes this frequency range attractive for a new generation of wireless communication systems - the so-called $\mathrm{THz}$ communications -, where high data rates can be achieved using bandwidths of several $10 \mathrm{~s}$ of $\mathrm{GHz}$ on one hand and applying low complex modulation schemes on the other hand.

To make this happen huge efforts in research are required for example in developing channel models, performing the corresponding channel measurements and in developing RF components. While first concepts for $\mathrm{THz}$ communications have been published already years ago, e. g. in $[2,3]$, in the more recent past a couple of promising hardware demonstrations have been realized, see e.g. $[4,5,6,7,8]$, relying on both electronic and photonic approaches in generating $\mathrm{THz}$ signals.

Furthermore, new standards have to be completed and regulatory aspects have to be addressed in order to secure the spectrum beyond $275 \mathrm{GHz}$ for $\mathrm{THz}$ communications.

In the following sections this paper provides an overview on the current status in the mentioned areas, namely channel measurements (section II), channel modeling (section III), hardware demonstrations (section IV), standardization and regulation (section V). Conclusions and an outlook are provided in section VI.

\section{Advanced Channel Measurements}

Whenever a new communication system is intended to operate in either a new frequency range or in a new environment or even both, the very first step in system design is always the determination of the typical propagation characteristics by measurements. Using carrier frequencies beyond $300 \mathrm{GHz}$ means the exploration a new frequency range for wireless communications and some of the above mentioned applications like intra-device communications or wireless links in data centers represent new operational environments on the same time.

In the literature three measurement techniques are reported, which are used to characterize the $\mathrm{THz}$ propagation channel: THz Time-Domain Spectroscopy (TDS), Vector Network Analysis (VNA) and Time-Domain Channel Sounding (CS).

TDS can be used for the characterization of scattering, reflection and transmission phenomena. For example, TDS was used to measure the influence of rough surfaces in scattering [9].

For the measurements of complete scenarios typically VNAs working in frequency domain are used [10,11]. Although a VNA provides a high dynamic range, its major drawbacks are its restriction to static environments and the time-consuming measurement procedure.

In order to measure dynamic scenarios and enable a less time-consuming measurement procedure a $\mathrm{CS}$ is a more favorable option. A CS based on a PN sequence operating at a carrier frequencies of 9,60 and $300 \mathrm{GHz}$ with $8 \mathrm{GHz}$ bandwidth is described in [12]. This CS has been also used recently to investigate the similarities of spatial channel characteristics at the above mentioned carrier frequencies in a lecture hall [13] and measure the effect of blocking my moving humans [31].

\section{Channel Modelling}

As it is the case for other frequency range a whole bunch of different models consisting of deterministic, stochastic and empirical approaches are available.

Ray-optical methods like ray tracing, which have proven their strength already at $60 \mathrm{GHz}$, e. g., are also applicable to higher frequencies especially in confined environments, like in indoor or intra-device situations. An example for a validation of ray tracing at $300 \mathrm{GHz}$ in indoor environments can be found in [14], e.g.. For intra-device communication printed circuit boards (PCB) are common scattering objects, where the size of the scattering objects are partially in the order of a wave length. A semi-empirical model is described in [15], which is dedicated to specifically characterize the scattering from PCB boards and which can be integrated into a ray tracing method..

For the performance evaluation of system proposals in a standardization process, where different partners shall have the possibility to run simulations in a similar way, deterministic models are not well-suited. Instead stochastic 
models are a better choice allowing the generation of a representative large number of channel representations in a random but still transparent way. These models can be either derived from measurements or ray-tracing simulations. For example the stochastic models for indoor environments, kiosk downloading, data centers and intra-device communications provided in $[16,17,18]$ have been derived using ray-tracing.

The Channel Modeling Document CMD [17] developed during the standardization of IEEE Std. 802.15.3d [22] includes also a set of empirical models for the description of atmospheric attenuation and weather effects, which are relevant at link distances of several $100 \mathrm{~m}$ meters, e. g. for a backhaul/ fronthaul application.

\section{HARDWARE DEMONSTRATIONS}

In the recent past many research groups all over the globe have made significant progress in developing RF front ends and antennas enabling wireless data transmission at $300 \mathrm{GHz}$ and beyond. Whereas at the receiving end of a link electronic approaches using mostly Schottky-Barrier Diodes are used, for the generation of the signals at the transmitting end both photonic [7,8] and electronic [4,19] approaches have been applied. Wireless data rates of $100 \mathrm{Gbit} / \mathrm{s}$ and beyond have been demonstrated, see e. g. [5].

Apart from potentially lower phase noise photonic approaches provide also the perspective of seamless fiberwireless integration. Such an approach has been pursued in the EU-Horizon2020 project iBROW [20] based on Resonant Tunneling Diodes (RTD). In the same project a compact solution has been presented, where a planar bow-tie antenna has been integrated on a chip using resonant tunneling diodes for the signal generation. [21].

In order to mitigate the high path loss, at least for longer distances high gain antennas may be used, which require electronic beam steering. This has been successfully demonstrated in [23] using a phased-array consisting of horn antennas allowing to steer a beam in azimuth direction within $\pm 20^{\circ}$ relative to the main direction. Despite this proof-ofconcept still a couple of challenges remain wrt beam steering: The small wave length makes it challenging to realize antenna arrays with a sufficiently high sidelobe suppression and device discovery under the regime of high-gain antennas require novel approaches like multi-frequency scanning [13]. Also beam tracking for moving users is demanding with high-gain antennas. A first simulation-based approach to solve the latter challenge exploiting measured statistics of user movements and applying Bayesian inference has been presented in [24].

\section{STANDARDISATION AND REGULATION}

In October 2017 Std. IEEE 802.15.3d-2017 has been published as the first wireless communication standard operating at carrier frequencies around $300 \mathrm{GHz}$ [22]. The standard is targeting fixed point-to-point links, where the positions of the antennas are known and the operational conditions allow the application of simplified multiple access schemes. The applications at which this standard is targeting are intra-device communication, kiosk downloading, complementary links in data centers and backhaul/fronthaul links. The key characteristics of the standard are as follows:
- The standard provids a new physical layer at 300 $\mathrm{GHz}$ and is an amendment to Std. IEEE 802.15.32016 [25] further amended by IEEE 802.15.3e$2017[26]$.

- $\quad 8$ different channel bandwidths of up to $69.12 \mathrm{GHz}$ realized as multiples of $2.16 \mathrm{GHz}$ have been defined.

- Two physical layer modes - a single carrier mode with BPSK, QPSK, 8-PSK, 8-APSK, 16-QAM, 64 QAM and an OOK mode - have been defined

- 3 channel coding schemes have been defined: a 14/15-rate LDPC $(1440,1344)$, a 11/14-rate LDPC $(1440,1056)$ and a 11/14-rate $\mathrm{RS}(240,224)$-code. The latter does not require soft decision information and is therefore specifically useful to the OOK mode which is the favorable scheme for solutions using RTDs.

Performance evaluations at link level done by software simulations [27] have revealed, that for backhaul/fronthaul applications distances of up to $453 \mathrm{~m}$ can be achieved for a data rate of $100 \mathrm{Gbit} / \mathrm{s}$. The underlying assumptions for this simulation are the use $40 \mathrm{dBi}$ gain antennas at both ends of the link, $1 \mathrm{~W}$ output power at the transmitter assuming the use of a traveling wave tube amplifier and an additional atmospheric attenuation of $20 \mathrm{~dB}$ derived using the methods from [17] for a heavy rain in a tropical city. At the other three applications the link distances are much smaller, which reduces the requirements wrt to the transmitter output power and the antenna gains. For example in intra-device environments with link distances of only a few centimeters, the use of transmitters with an output power of $0 \mathrm{dBm}$ and antennas with a gain of $6 \mathrm{dBi}$ are sufficient.

At IEEE 802.15 the THz Interest Group is still active and is working towards the identification of further additional applications, which would require a further amendment of the current standard.

In terms of spectrum for $\mathrm{THz}$ communications the radio regulations allow the use of spectrum beyond $275 \mathrm{GHz}$, if passive services like radio astronomy and earth-exploration satellite services are protected from harmful interference according to footnote 5.565 [28]. In order to enable the use of the required large chunks of spectrum of several $10 \mathrm{~s}$ of $\mathrm{GHz}$ per wireless connection sharing of $\mathrm{THz}$ Communications with the above mentioned passive services is a pre-requisite. First investigations of potential interference between active and passive services have been carried out by Priebe at. al [29] indicating the chances and challenges for such a sharing scenario. The World Radio Conference (WRC) 2015 has put the consideration of identification of spectrum for land-mobile and fixed active services in the range of $275 \mathrm{GHz}$ to $450 \mathrm{GHz}$ while maintaining protection of the passive services identified in the existing footnote 5.565 under agenda item 1.15 at the upcoming World Radio Conference (WRC) 2019 [30]. In this process ITU-R will identify technical and operational characteristics, study spectrum needs for $\mathrm{THz}$ communications, develop propagation models, conduct sharing studies with the passive services and identify candidate frequency bands. 


\section{CONCLUSIONS AND OUTLOOK}

Frequency bands beyond $275 \mathrm{GHz}$ offer a huge potential to implement wireless communication systems with data rates targeting $100 \mathrm{Gbit} / \mathrm{s}$ and beyond. This paper has provided a brief overview on the current status of the development of $\mathrm{THz}$ Communication systems focusing on recent results on advanced channel characterization at $300 \mathrm{GHz}$, hardware demonstrators operating in this frequency range, the past and current activities at IEEE 802 and the WRC 2019 preparations.

Within the European Horizon 2020 calls ICT-09-2017 "Networking research beyond 5G" and EUJ-02-2018 Specific challenges of networks beyond" totally seven projects dealing completely or partially with $\mathrm{THz}$ communications have been granted [32]. The targets envisaged by these projects are amongst others demonstrators and components for wireless data centers and backhaul/fronthaul links as well as next generation channel coding and wireless-optical integration enabling Tbit/s data rates.

\section{ACKNOWLEDGEMENT}

Parts of the research described in this paper has been carried out with the project iBROW (Grant Agreement No. 645369) supported by the EU Framework Program for Research and Innovation Horizon 2020.

\section{REFERENCES}

[1] T. Kürner et. al, "Applications Requirement Document (ARD)", DCN: 1514-0304-16-003d, IEEE 802.15 TG3d, May 2015 , https://mentor.ieee.org/802.15/documents

[2] R. Piesiewicz, T. Kleine-Ostmann, N. Krumbholz, D. Mittleman, M. Koch, J. Schöbel and T. Kürner: Short-Range Ultra Broadband Terahertz Communications: Concept and Perspectives, IEEE Antennas \& Propagation Magazine 49, 24-39 (2007).

[3] T. Kleine-Ostmann and T. Nagatsuma: A Review on Terahertz Communications Research, J Infrared Milli Terahz Waves 32, 143-171 (2011).

[4] J. Antes et al., "Transmission of an 8-PSK modulated 30 Gbit/s signal using an MMIC-based $240 \mathrm{GHz}$ wireless link," IEEE MTT-S Int. Microwave Symp. Digest (IMS) (2013)

[5] S. Koenig et al., "Wireless sub-THz communication system with high data rate," Nature Photonics 7, pp. 977-981 (2013).

[6] I. Kallfass, F. Boes et al. "64 Gbit/s Transmission over $850 \mathrm{~m}$ fixed wireless link at $240 \mathrm{GHz}$ carrier frequency," J. Infrared Milli. Terahz Waves 36 , pp. 221-233 (2015).

[7] T. Nagatsuma et al., "Terahertz wireless communications based on photonics technologies," Opt. Express 21, pp. 477487 (2013).

[8] T. Nagatsuma, G. Ducournau, "Advances in terahertz communications accelerated by photonics," Nature Photonics, 10, pp. 371379 (2016)

[9] Piesiewicz, R. Jansen, C; Mittleman, D; Kleine-Ostmann, T; Koch, M. Kürner, T.; Scattering analysis for the modeling of $\mathrm{THz}$ communication systems; IEEE Trans. on Antennas and Propagation, Vol. 55, No. 11 November 2007, pp.3002-3009

[10] M. Jacob, S. Priebe, R. Dickhoff, T. Kleine-Ostmann, T. Kürner: Diffraction in $\mathrm{mm}$ and sub-mm Wave Indoor Propagation Channels. IEEE Transactions on Microwave Theory and Techniques, Vol. 60, No. 3, pp. 833844,2012

[11] S. Kim and A. Zajić, "Characterization of 300-GHz Wireless Channel on a Computer Motherboard," in IEEE Transactions on Antennas and Propagation, vol. 64, no. 12, pp. 5411-5423, Dec. 2016

[12] S. Rey, J. M. Eckhardt, B. Peng, K. Guan and T. Kürner, "Channel sounding techniques for applications in $\mathrm{THz}$ communications: A first correlation based channel sounder for ultra-wideband dynamic channe measurements at $300 \mathrm{GHz}$," in 9th International Congress on Ultra Modern
Telecommunications and Control Systems and Workshops (ICUMT), Munich, 2017.

[13] B. Peng, K. Guan, S. Rey, T. Kürner, Two-Step Angle-of-Arrival Estimation for Terahertz Communications Based on Correlation of PowerAngular Spectra in Frequency, Proc. European Conference on Antennas and Propagation EuCAP 2018, London, March 2018, electronic publication, 5 pages

[14] S. Priebe , M. Kannicht, M. Jacob and T. Kürner, Ultra Broadband Indoor Channel Measurements and Calibrated Ray Tracing Propagation Modeling at $\mathrm{THz}$ Frequencies, Journal of Communications and Networks, December 2013, Vol. 15, No. 6, pp.547-558,

[15] Fricke A, Achir M, Le Bars P, Kürner T. A model for the reflection of terahertz signals from printed circuit board surfaces. International Journal of Microwave and Wireless Technologies

[16] Priebe, S.; Kürner, T.: Stochastic Modelling of $\mathrm{THz}$ Indoor Radio Channels. IEEE Transactions on Wireless Communications, Vol. 12, No. 6, pp. 44454455,2013

[17] A. Fricke, B. Peng, T. Kürner et al., Channel Modeling Document (CMD): https://mentor.ieee.org/802.15/dcn/14/15-14-0310-18-003d-channelmodeling-document.docx

[18] D. He et al., "Stochastic Channel Modeling for Kiosk Applications in the Terahertz Band," in IEEE Transactions on Terahertz Science and Technology, vol. 7, no. 5, pp. 502-513, Sept. 2017

[19] I. Kallfass, I. Dan, S. Rey, P. Harati, J. Antes, A. Tessmann, S. Wagner, M. Kuri, R. Weber, H. Massler, A. Leuther, T. Merkle, T. Kürner, "Towards mmic-based $300 \mathrm{ghz}$ indoor wireless communication systems," Trans. Institute of Electronics, Information and Communication Engineers IEICE, vol. E98-C, no. 12, pp. 1081-1090, Dec. 2015

[20] http://ibrow-project.eu/

[21] K. H. Alharbi et al., Diced and grounded broadband bow-tie antenna with tuning stub for resonant tunnelling diode terahertz oscillators, IET Microwaves, Antennas \& Propagation(2017),11(3):310

[22] IEEE Standard for High Data Rate Wireless Multi-Media Networks-Amendment 2: $100 \mathrm{~Gb} / \mathrm{s}$ Wireless Switched Point-to-Point Physical Layer," in IEEE Std 802.15.3d-2017 (Amendment to IEEE Std 802.15.3-2016 as amended by IEEE Std 802.15.3e-2017), vol., no., pp.1-55, Oct. 182017

[23] S. Rey, D. Ulm, T. Kleine-Ostmann, and T. Kürner, "Performance evaluation of a first phased array operating at $300 \mathrm{GHz}$ with horn elements," in Proceedings of the 11th European Conference on Antennas and Propagation (EUCAP), pp. 1629-1633, 2017.

[24] B. Peng, and T. Kürner. "Three dimensional angle of arrival estimation in dynamic indoor terahertz channels using forward-backward algorithm. ", IEEE Transactions on Vehicular Technology, Vol. 66, No. 5, May 2017, pp. 37983811

[25] IEEE Standard for High Data Rate Wireless Multi-Media Networks," in IEEE Std 802.15.3-2016 (Revision of IEEE Std 802.15.3-2003 , vol., no., pp.1-510, July 252016

[26] IEEE Standard for High Data Rate Wireless Multi-Media Networks-Amendment 1: High-Rate Close Proximity Point-to-Point Communications," in IEEE Std 802.15.3e-2017 (Amendment to IEEE Std 802.15.3-2016), vol., no., pp.1-178, June 72017

[27] https://mentor.ieee.org/802.15/dcn/17/15-17-0039-04-003d-summary-ofresults-from-tg3d-link-level-simulations.pdf

[28] ITU-R: Radio Regulations 2016; http://www.itu.int/pub/R-REG-RR

[29] RESOLUTION 767 (WRC-15): Studies towards an identification for use by administrations for land mobile and fixed services applications operating in the frequency range 275-450GHz: https://www.itu.int/en/ITU-R/studygroups/rcpm/Pages/wrc-19-studies.aspx

[30] S. Priebe et al., "Interference Investigations of Active Communications and Passive Earth Exploration Services in the THz Frequency Range," IEEE Trans. on Terahertz Science and Technology, 2, 5, pp. 525537 (2012).

[31] B. Peng et al, "Statistical Characteristics Study of Human Blockage Effect in Future Indoor Millimeter and Sub-millimeter Wave Wireless Communications", Proc. IEEE Vehicular Technology Conference VTC 2018 Spring, Porto/Portugal, June 2018

[32] http://terapod-project.eu/links/ict-09-2017-cluster/ 